\title{
Phase-Transfer Catalyzed Alkylation of Hydantoin and 5-Methyl-5-Phenylhydantoin
}

\author{
Tariq R. Sobahi*, Mohamed A. Hassan, \\ Huissen A. Moustafa and Nahed O. Bawaked \\ Department of Chemistry, Faculty of Science, \\ King Abdulaziz University, \\ P.O. Box 80203, Jeddah 21589, Saudi Arabia \\ *drtariq_s@hotmail.com
}

\begin{abstract}
PTC-alkylation of hydantoin (1a) and 5-methyl-5-phenylhydantoin $(\mathbf{1 b})$ by different organohalogen reagents at $25^{\circ} \mathrm{C}$ in the presence of tetrabutylammonium bromide as catalyst has been investigated either in the absence or presence of $\mathrm{CS}_{2}$. This work is aiming to study the comparative reactivity of $\mathrm{N}$ - versus $\mathrm{O}$ - of hydantoins toward alkylation and cycloalkylation. In all cases N3 monoalkylation or N1 and N3 dialkylation are the main products. The structures of alkylhydantoins have been established by IR, NMR, mass spectral data and elemental analysis.
\end{abstract}

Keywords: Phase-transfer catalysis (PTC), alkylation, cycloalkylation, hydantoin, 5-methyl-5-phenylhydantoin, tetrabutylammonium bromide.

\section{Introduction}

Phase-transfer catalysis (PTC) is one of the promising methods in organic synthesis of specialty chemicals. In the last 20 years, a steadily increasing number of published papers and patents dealing with phase transfer catalysis topics and their applications. PTC is not merely important for substitution reactions but, nowadays, it is being extensively applied in polymer chemistry, heterocyclic chemistry, organometallic, agrochemicals, dyes, flavors, perfumes and pharmaceutical manufacture ${ }^{[1-3]}$. 
The technique of PTC has, extensively, been applied in the organic synthesis via substitution, displacement, condensation, elimination, redox, polymerization and Ylide-mediated reactions. The most advantages of using PTC technique to synthesize organic chemicals, are the enhancement of the reaction rate, carrying out the reaction at moderate conditions, obtaining high selectivity of the main product with high conversion of the reactants ${ }^{[4,5]}$.

In continuation of our current research in the field of organic synthesis of some heterocyclic compounds via phase transfer catalysis conditions (PTC) ${ }^{[6-10]}$, we are aiming here to study the reactivity of N- vs O- alkylations of hydantoin (1a) and 5-methyl-5-phenylhydantoin (1b) in absence or presence of carbon disulphide.

Hydantoins are very important class of organic compounds with numerous pharmaceutical applications ${ }^{[11,12]}$. They have a wide biological activities such as integrins and kinases inhibitors ${ }^{[13,14]}$, anti-convulsants \& anti-epileptics ${ }^{[15]}$, fungicides $\&$ herbicides ${ }^{[16]}$, anti-bacterial $\&$ anti-mycobacterial ${ }^{[17]}$ and potent activity against the herpes simplex virus (HSV) ${ }^{[18]}$, human immunodeficiency virus $(\mathrm{HIV})^{[19]}$ and the leukemia subpanel ${ }^{[20]}$.

\section{Experimental}

All melting points reported are uncorrected. IR $(\mathrm{KBr})$ spectra were recorded using Perkin Elmer's spectrum RXIFT-IR spectrophotometer $\left(v\right.$ in $\left.\mathrm{cm}^{-1}\right)$. The NMR spectra were recorded on Bruker Avance DP $\times 400$ spectrometer, using $\mathrm{CDCl}_{3}$ as solvent and TMS as internal standard (chemical shifts in $\delta$ values in ppm). Elemental analysis was performed on Perkin Elmer 2400, series II microanalyzer. The mass spectra were recorded by Shimadzu GC-17A gas chromatograph QP-5000 mass spectrometer. Hydantoins (1a,b) are Aldrich products and they are used without any further purifications.

\section{General Procedure of PTC-Alkylation of Hydantoins (1a,b)}

\section{a) In the Absence of Carbon Disulphide}

In a $100 \mathrm{ml}$ conical flask, fitted with rubber stopper, a suspension of hydantoins $(\mathbf{1 a}, \mathbf{b})(0.01 \mathrm{~mol})$, anhydrous potassium carbonate $(0.02 \mathrm{~mol}, 2.76 \mathrm{~g})$ and tetrabutylammonium bromide (TBAB, $0.003 \mathrm{~mol}, 0.9 \mathrm{~g}$ ) in tetrahydrofuran (THF, $100 \mathrm{ml}$ ) were stirred at $25^{\circ} \mathrm{C}$ for $30 \mathrm{~min}$, then the organohalogen compound (alkylating agents) ( $0.03 \mathrm{~mol}$ for monohalogen compounds, and 0.01 mol for dihalogen reagents), namely, ethyl bromide, n-propyl bromide, n-butyl bromide, allyl bromide, benzyl bromide, ethyl bromoacetate, 1,2-dibromoethane 
or 1,3-dibromopropane was added. The reaction mixture was efficiently stirred at room temperature. The progress of the reaction was monitored by TLC during the entire reaction period. After the completion of the reaction, the organic layer was separated by filtration and the organic solvent was evaporated and the residue was crystallized from the proper solvent or separated by column chromatography using silica gel (80-120 mesh) and diethyl ether/petroleum ether $(2: 1)$ as eluent to separate the products from the unreacted hydantoins. On the other hand, the $\mathrm{K}_{2} \mathrm{CO}_{3}$ precipitate was dissolved in water $(100 \mathrm{ml})$ and acidified by dilute $\mathrm{HCl}(10 \%)$ to separate any acidic products, if any, but no acidic products were isolated by all alkylating agents. The results are listed in Table 1 .

\section{b) In the Presence of Carbon Disulphide}

In a round bottle flask $(100 \mathrm{ml})$ fitted with a water condenser, a suspension of hydantoins $(\mathbf{1 a}, \mathbf{b})(0.01 \mathrm{~mol})$, anhydrous $\mathrm{K}_{2} \mathrm{CO}_{3}(0.02 \mathrm{~mol}, 2.76 \mathrm{~g})$, tetrabutylammonium bromide (TBAB, $0.003 \mathrm{~mol}, 0.90 \mathrm{~g}$ ) and carbon disulphide (10 $\mathrm{ml}$ ) in tetrahydrofuran (THF, $100 \mathrm{ml}$ ) was added. The mixture was efficiently stirred at room temperature for $30 \mathrm{~min}$, then the organohalogen reagents $(0.05$ mol) were added and the reaction mixture vigorously stirred at $25^{\circ} \mathrm{C}$. The progress of the reaction was monitored by TLC over the entire reaction period. After completion of the reaction, the organic layer was separated by filtration and the solvent was evaporated. The products were separated by column chromatography using silica gel (80-120 mesh) and diethyl ether/petroleum ether (2:1) as eluent to separate the products from the unreacted hydantoins. The solid residue was crystallized from appropriate solvent. The results are listed in Table 1.

\section{Results and Discussion}

The approach reported here is an extension and continuation of our interest in alkylation of some heterocycles under phase-transfer catalysis (PTC) conditions ${ }^{[6-10]}$. This work is aiming to study the phase-transfer catalyzed alkylation of hydantoin (1a) and 5-methyl-5-phenylhydantoin (1b) and the comparative reactivity towards $\mathrm{N}-v_{s} \mathrm{O}$ - upon treatment with different organohalogen reagents in liquid/solid in the presence of tetrabutylammonium bromide (TBAB) as a catalyst in the absence or presence of $\mathrm{CS}_{2}$ at $25^{\circ} \mathrm{C}$.

On the other hand, we are aiming to synthesize new hydantoin derivatives which might have an expected biological activity in addition to the well known biological, pharmacological and medicinal applications of hydantoins ${ }^{[11-20]}$.

5-Methyl-5-phenylhydantoin (1b) may exist in different tautomeric structures I-VII (Scheme 1). 
Table 1. The physical data of alkylated products (2-4).

\begin{tabular}{|c|c|c|c|c|c|c|c|}
\hline \multirow{2}{*}{$\begin{array}{c}\text { Compd. } \\
\text { no. }\end{array}$} & \multirow{2}{*}{$\begin{array}{c}\text { Reaction } \\
\text { period } \\
\text { t (h) }\end{array}$} & \multirow{2}{*}{$\begin{array}{l}\text { M. formula } \\
\text { (m. wt.) }\end{array}$} & \multirow{2}{*}{$\begin{array}{l}\text { m.p. } .^{\circ} \mathrm{C} \\
\text { (colour) }\end{array}$} & \multirow{2}{*}{$\begin{array}{l}\text { Solvent }^{\#} \\
\text { Cryston } \\
\text { (yield \%) }\end{array}$} & \multicolumn{3}{|c|}{$\begin{array}{l}\text { Elemental analysis } \\
\text { cal.c/found \% }\end{array}$} \\
\hline & & & & & $\mathrm{C}$ & $\mathbf{H}$ & $\mathbf{N}$ \\
\hline \multirow[t]{2}{*}{$2 a$} & \multirow[t]{2}{*}{7} & \multirow{2}{*}{$\begin{array}{l}\mathrm{C}_{5} \mathrm{H}_{8} \mathrm{~N}_{2} \mathrm{O}_{2} \\
(128.13))\end{array}$} & $102-105$ & P.E. $60-80$ & 46.87 & 6.29 & 21.86 \\
\hline & & & (white) & (73) & 46.73 & 6.32 & 21.98 \\
\hline \multirow[t]{2}{*}{$2 \mathrm{~b}$} & \multirow[t]{2}{*}{6} & \multirow{2}{*}{$\begin{array}{c}\mathrm{C}_{6} \mathrm{H}_{10} \mathrm{~N}_{2} \mathrm{O}_{2} \\
(142.16)\end{array}$} & $86-88$ & P.E. $60-80$. & 50.69 & 7.09 & 19.71 \\
\hline & & & (white) & (67) & 50.55 & 6.90 & 19.88 \\
\hline \multirow[t]{2}{*}{$2 c$} & \multirow[t]{2}{*}{9} & \multirow{2}{*}{$\begin{array}{c}\mathrm{C}_{7} \mathrm{H}_{12} \mathrm{~N}_{2} \mathrm{O}_{2} \\
(156.18)\end{array}$} & $97-99$ & P.E. $60-80$. & 53.83 & 7.74 & 17.94 \\
\hline & & & (white) & (65) & 53.57 & 7.76 & 18.08 \\
\hline \multirow[t]{2}{*}{ 2d } & \multirow[t]{2}{*}{24} & \multirow{2}{*}{$\begin{array}{c}\mathrm{C}_{6} \mathrm{H}_{8} \mathrm{~N}_{2} \mathrm{O}_{2} \\
(140.14)\end{array}$} & $75-77$ & P.E. $60-80$. & 51.42 & 5.75 & 19.99 \\
\hline & & & (white) & (45) & 51.27 & 5.71 & 19.92 \\
\hline \multirow[t]{2}{*}{$2 e$} & \multirow[t]{2}{*}{2} & \multirow{2}{*}{$\begin{array}{c}\mathrm{C}_{10} \mathrm{H}_{10} \mathrm{~N}_{2} \mathrm{O}_{2} \\
(190.20)\end{array}$} & 137 & P.E. / B & 63.15 & 5.30 & 14.73 \\
\hline & & & (white) & $(61)$ & 62.88 & 5.35 & 14.88 \\
\hline \multirow[t]{2}{*}{$2 f$} & \multirow[t]{2}{*}{7} & \multirow{2}{*}{$\begin{array}{c}\mathrm{C}_{5} \mathrm{H}_{7} \mathrm{~N}_{2} \mathrm{O}_{2} \mathrm{Br} \\
(207.03)\end{array}$} & $136-138$ & P.E. / B & 29.01 & 3.41 & 13.53 \\
\hline & & & (white) & (80) & 28.68 & 3.39 & 13.45 \\
\hline $2 \mathrm{~g}$ & 7 & $\mathrm{C}_{6} \mathrm{H}_{9} \mathrm{~N}_{2} \mathrm{O}_{2} \mathrm{Br}$ & $118-120$ & P.E. $80-100$ & 32.60 & 4.10 & 12.67 \\
\hline & & $(221.06)$ & (white) & $(91)$ & 32.47 & 4.14 & 12.76 \\
\hline $2 \mathrm{~h}$ & 24 & $\mathrm{C}_{11} \mathrm{H}_{16} \mathrm{~N}_{2} \mathrm{O}_{6}$ & Oil & Ether / P.E. (1:1) & 48.53 & 5.93 & 10.29 \\
\hline & & $(272.26)$ & (yellow) & $(68)$ & 48.38 & 5.87 & 10.38 \\
\hline $2 \mathrm{i}$ & 1 & $\mathrm{C}_{12} \mathrm{H}_{14} \mathrm{~N}_{2} \mathrm{O}_{2}$ & 109 & P.E. / B & 66.04 & 6.47 & 12.84 \\
\hline & & $(218.25)$ & & $(68)$ & 65.83 & 6.39 & 12.96 \\
\hline $2 \mathrm{j}$ & 0.5 & $\mathrm{C}_{13} \mathrm{H}_{16} \mathrm{~N}_{2} \mathrm{O}_{2}$ & 100 & P.E. & 67.22 & 6.94 & 12.06 \\
\hline & & $(232.28)$ & & $(65)$ & 66.83 & 6.83 & 12.18 \\
\hline $2 \mathrm{k}$ & 0.5 & $\mathrm{C}_{14} \mathrm{H}_{18} \mathrm{~N}_{2} \mathrm{O}_{2}$ & 91 & P.E. & 68.27 & 7.37 & 11.37 \\
\hline & & $(246.31)$ & & (61) & 68.05 & 7.34 & 11.35 \\
\hline 21 & 3 & $\mathrm{C}_{13} \mathrm{H}_{14} \mathrm{~N}_{2} \mathrm{O}_{2}$ & $76-8$ & P.E. & 67.81 & 6.13 & 12.17 \\
\hline & & $(230.27)$ & & $(68)$ & 67.71 & 6.16 & 12.23 \\
\hline $2 m$ & 10 & $\mathrm{C}_{12} \mathrm{H}_{13} \mathrm{~N}_{2} \mathrm{O}_{2} \mathrm{Br}$ & 261 & E & 48.50 & 4.41 & 9.43 \\
\hline & & $(297.15)$ & & $(31.1)$ & 48.41 & 4.37 & 9.52 \\
\hline $2 n$ & 5 & $\mathrm{C}_{13} \mathrm{H}_{15} \mathrm{~N}_{2} \mathrm{O}_{2} \mathrm{Br}$ & 115 & P.E. & 50.18 & 4.86 & 9.00 \\
\hline & & $(311.18)$ & & (58) & 50.02 & 4.84 & 9.17 \\
\hline 20 & 2 & $\mathrm{C}_{24} \mathrm{H}_{22} \mathrm{~N}_{2} \mathrm{O}_{2}$ & 115 & $\mathrm{E}$ & 77.81 & 5.99 & 7.56 \\
\hline & & $(370.45)$ & & $(90)$ & 77.69 & 5.97 & 7.63 \\
\hline $2 p$ & 3 & $\mathrm{C}_{18} \mathrm{H}_{22} \mathrm{~N}_{2} \mathrm{O}_{6}$ & oil & E / P.E. & 59.66 & 6.12 & 7.73 \\
\hline & & $(362.38)$ & & (eluent) (75) & 59.52 & 6.13 & 7.82 \\
\hline 3 & 3 & $\mathrm{C}_{13} \mathrm{H}_{14} \mathrm{~N}_{2} \mathrm{O}_{2}$ & $119-21$ & P.E. / B & 67.81 & 6.13 & 12.17 \\
\hline & & $(230.27)$ & & $(60)$ & 67.67 & 6.15 & 12.27 \\
\hline $4 a$ & 2 & $\mathrm{C}_{16} \mathrm{H}_{28} \mathrm{~N}_{2} \mathrm{O}_{2} \mathrm{~S}_{2}$ & $74-76$ & P.E. & 55.78 & 8.19 & 8.13 \\
\hline & & $(344.54)$ & (yellow) & $(32)$ & 55.64 & 8.21 & 8.21 \\
\hline $4 b$ & 24 & $\mathrm{C}_{16} \mathrm{H}_{20} \mathrm{~N}_{2} \mathrm{O}_{2} \mathrm{~S}_{2}$ & $86-88$ & Ether / P.E.(1:2) & 57.11 & 5.99 & 8.33 \\
\hline & & $(336.48)$ & (yellow) & $(38)$ & 56.95 & 5.97 & 8.40 \\
\hline $4 c$ & 24 & $\mathrm{C}_{16} \mathrm{H}_{22} \mathrm{~N}_{2} \mathrm{O}_{8} \mathrm{~S}_{2}$ & Oil & Ether / P.E. (1:1) & 44.23 & 5.10 & 6.45 \\
\hline & & $(434.49)$ & (yellow) & $(59)$ & 44.07 & 5.13 & 6.53 \\
\hline
\end{tabular}

P.E. $=$ petroleum ether, $\mathrm{E}=$ ethanol, $\mathrm{B}=$ benzene 


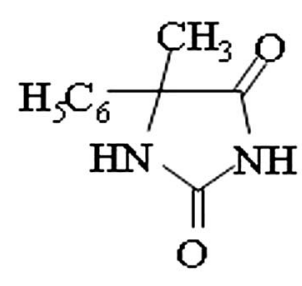

I

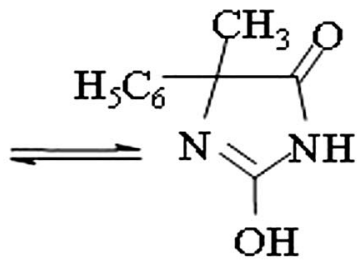

II

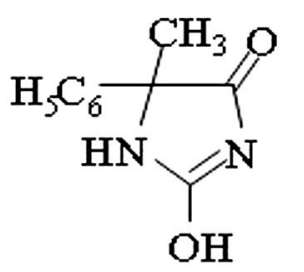

III

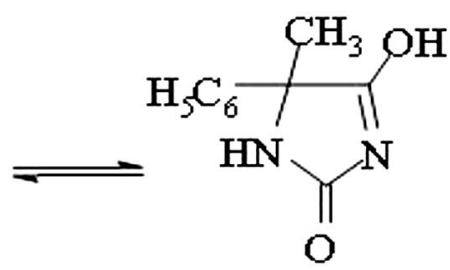

TV

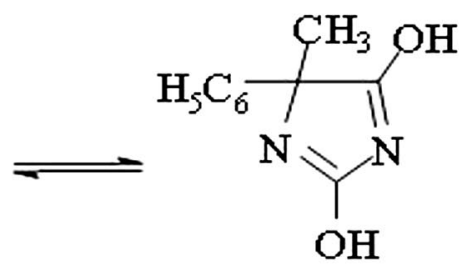

$\mathrm{V}$

Scheme 1

The structure of hydantoin and 1-mono or 1,5-disubstituted hydantoins were investigated ${ }^{[21]}$ by one and two dimensional ${ }^{1} \mathrm{H}$ and ${ }^{13} \mathrm{CNMR}$ techniques, which proved the existence of hydantoins, predominantly, in the lactam form (I). Also, the IR spectrum of hydantoin (1a) displayed an absorption bands at $v$ (in $\left.\mathrm{cm}^{-1}\right)$ : $1706(\mathrm{C}=\mathrm{O}), 1774(\mathrm{C}=\mathrm{O}), 2765(\mathrm{CH}), 3062(\mathrm{CH}), 3146(\mathrm{NH})$ and $3258(\mathrm{NH})$, while the ${ }^{1} \mathrm{HNMR}$ (pyridine- $\mathrm{d}_{5}$ ) displayed signals at $\delta$ (in ppm): $5.28\left(\mathrm{~s}, 2 \mathrm{H}, \mathrm{C}_{2}\right), 10.00(\mathrm{~s}, 1 \mathrm{H}, \mathrm{N} 1-\underline{\mathrm{H}})$ and $13.53(\mathrm{~s}, 1 \mathrm{H}, \mathrm{N} 3-\underline{\mathrm{H}})$.

Treatment of hydantoins (1a,b) with ethyl bromide, n-propyl bromide, n-butyl bromide, allyl bromide, benzyl bromide (1:3 molar ratio), 1,2-dibromoethane and 1,3-dibromopropane (1:1 molar ratio) in tetrahydrofuran (THF) and anhydrous potassium carbonate $\left(\mathrm{K}_{2} \mathrm{CO}_{3}\right)$ as liquid/solid phases and in the presence of tetrabutylammonium bromide as catalyst at $25^{\circ} \mathrm{C}$ and absence of $\mathrm{CS}_{2}$ with efficient stirring gives after a short reaction period $(\mathrm{t})$, a promising yield of 3-alkylhydantions (3-alkylimidozolidine-2,4-diones) (2a-g) or 3-alkyl5-methyl-5-phenyl- hydantion (2i-n) in good yield, respectively (Scheme 2). The alkylation occurs, exclusively, at nitrogen in position-3 (N3) to give the mono-alkylated product (2a-g \& 2i-n), (Scheme 2), except hydantoin (1a) was alkylated by ethyl bromoacetate to give diethyl 2,2'(2,4-dioxoimidazoliden-1,3diyl) diacetate (2h) as pale yellow oil via $\mathrm{N} 1$ and $\mathrm{N} 3$ dialkylation, while 5-methyl-5-phenylhydantoin (1b) was alkylated by benzyl bromide or ethyl bromoacetate as a highly reactive organohalogen reagent afforded, 1,3dibenzyl-5-methyl-5-phenylhydantoin (2o) or diethyl 2,2'(5-methyl-5-phenyl2,4-dioxoimidazoliden-1,3-diyl) diacetate (2p) via N1, N3 simultaneous dial- 
kylation (Scheme 2). Meanwhile, treatment of 5-methyl-5-phenyl- hydantoin (1b) with 1,3-dibromopropane (2:1 molar ratio) under the same PTC reaction conditions underwent cycloalkylation via $\mathrm{N} 1$ and $\mathrm{N} 3$, simultaneous dialkylation to give, 7-methyl-7-phenyl-1,5-diazabicyclo[3.2.1] octane-6,8-dione (3), (Scheme 2).

The structure of mono and dialkylated hydantoins (2a-g and 3) has been established by spectral data (Table 2) and elemental analysis (Table 1).

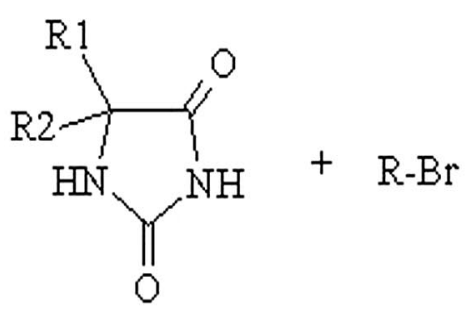

(1)

a) $\mathrm{R}^{1}=\mathrm{R}^{2}=\mathrm{H}$

b) $\mathrm{R}^{\mathrm{l}}=\mathrm{Me}, \mathrm{R}^{2}=\mathrm{Ph}$

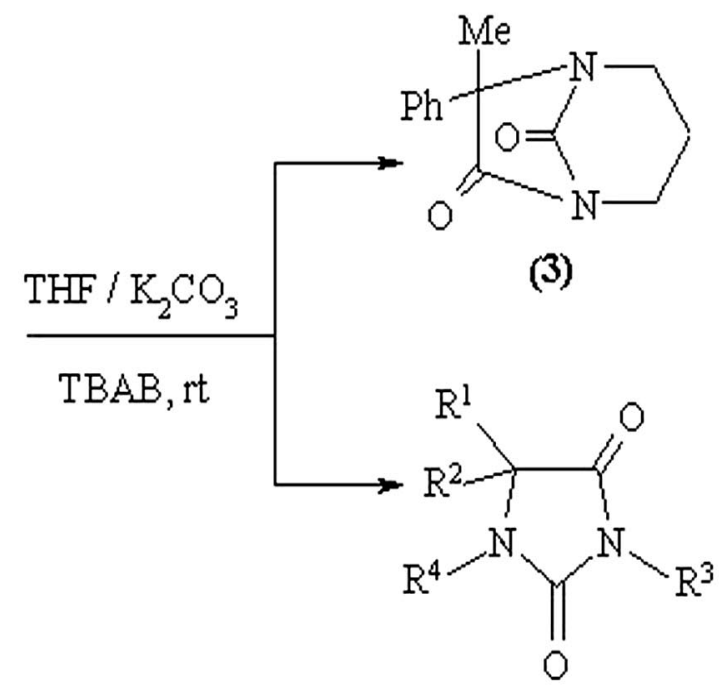

(2)

\begin{tabular}{c|cccc}
$\mathbf{2}$ & $\mathrm{R}^{1}$ & $\mathrm{R}^{2}$ & \multicolumn{1}{c}{$\mathrm{R}^{3}$} & $\mathrm{R}^{4}$ \\
\hline $\mathbf{a}$ & $\mathrm{H}$ & $\mathrm{H}$ & $\mathrm{C}_{2} \mathrm{H}_{5}$ & $\mathrm{H}$ \\
$\mathbf{b}$ & $\mathrm{H}$ & $\mathrm{H}$ & $\mathrm{n}-\mathrm{C}_{3} \mathrm{H}_{7}$ & $\mathrm{H}$ \\
$\mathbf{c}$ & $\mathrm{H}$ & $\mathrm{H}$ & $\mathrm{n}^{-} \mathrm{C}_{4} \mathrm{H}_{9}$ & $\mathrm{H}$ \\
$\mathbf{d}$ & $\mathrm{H}$ & $\mathrm{H}$ & $\mathrm{CH}_{2} \mathrm{CH}=\mathrm{CH}_{2}$ & $\mathrm{H}$ \\
$\mathbf{e}$ & $\mathrm{H}$ & $\mathrm{H}$ & $\mathrm{C}_{6} \mathrm{H}_{5} \mathrm{CH}_{2}$ & $\mathrm{H}$ \\
$\mathbf{f}$ & $\mathrm{H}$ & $\mathrm{H}$ & $\left(\mathrm{CH}_{2}\right)_{2} \mathrm{Br}$ & $\mathrm{H}$ \\
$\mathbf{g}$ & $\mathrm{H}$ & $\mathrm{H}$ & $\left(\mathrm{CH}_{2}\right)_{3} \mathrm{Br}$ & $\mathrm{H}$ \\
$\mathbf{h}$ & $\mathrm{H}$ & $\mathrm{H}$ & $\mathrm{CH}_{2} \mathrm{CO}_{2} \mathrm{Et}$ & $\mathrm{CH}_{2} \mathrm{CO}_{2} \mathrm{Et}$ \\
$\mathbf{i}$ & $\mathrm{Me}$ & $\mathrm{Ph}$ & $\mathrm{C}_{2} \mathrm{H}_{5}$ & $\mathrm{H}$ \\
$\mathbf{j}$ & $\mathrm{Me}$ & $\mathrm{Ph}$ & $\mathrm{n}_{5}-\mathrm{C}_{3} \mathrm{H}_{7}$ & $\mathrm{H}$ \\
$\mathbf{k}$ & $\mathrm{Me}$ & $\mathrm{Ph}$ & $\mathrm{n}_{-}-\mathrm{C}_{4} \mathrm{H}_{9}$ & $\mathrm{H}$ \\
$\mathbf{I}$ & $\mathrm{Me}$ & $\mathrm{Ph}$ & $\mathrm{CH}_{2} \mathrm{CH}_{3} \mathrm{CH}_{2}$ & $\mathrm{H}$ \\
$\mathbf{m}$ & $\mathrm{Me}$ & $\mathrm{Ph}$ & $\left(\mathrm{CH}_{2}\right)_{2} \mathrm{Br}$ & $\mathrm{H}$ \\
$\mathbf{n}$ & $\mathrm{Me}$ & $\mathrm{Ph}$ & $\left(\mathrm{CH}_{2}\right)_{3} \mathrm{Br}$ & $\mathrm{H}$ \\
$\mathbf{o}$ & $\mathrm{Me}$ & $\mathrm{Ph}$ & $\mathrm{C}_{6} \mathrm{H}_{5} \mathrm{CH}_{2}$ & $\mathrm{C}_{6} \mathrm{H}_{5} \mathrm{CH}_{2}$ \\
$\mathbf{P}$ & $\mathrm{Me}$ & $\mathrm{Ph}$ & $\mathrm{CH}_{2} \mathrm{CO}_{2} \mathrm{Et}$ & $\mathrm{CH}_{2} \mathrm{CO}_{2} \mathrm{Et}$
\end{tabular}

Scheme 2 


\begin{tabular}{|c|c|c|c|c|c|}
\hline 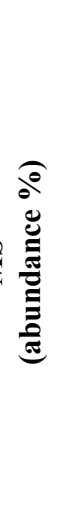 & 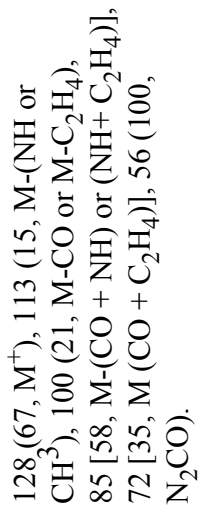 & 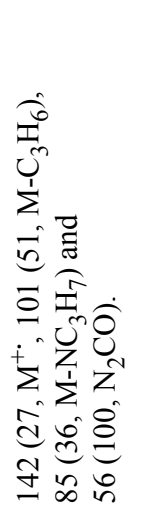 & 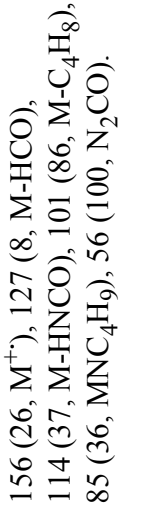 & 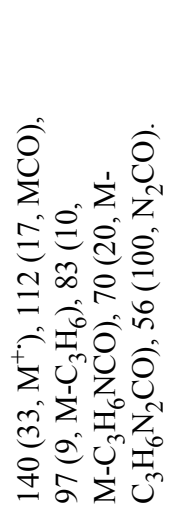 & 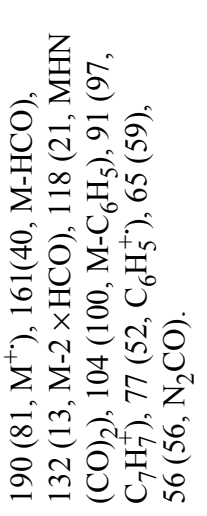 \\
\hline$\widehat{\underline{0}}$ & 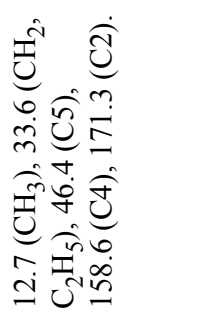 & 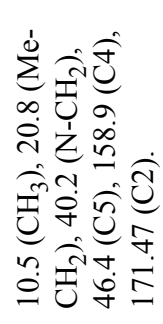 & 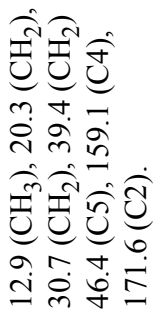 & & 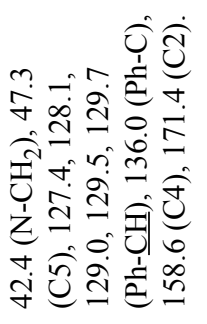 \\
\hline $\begin{array}{l}\widehat{E} \\
0 \\
0 \\
0 \\
0 \\
0 \\
0\end{array}$ & 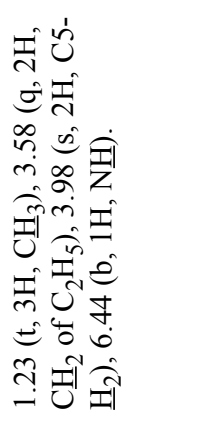 & 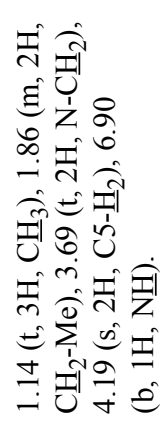 & 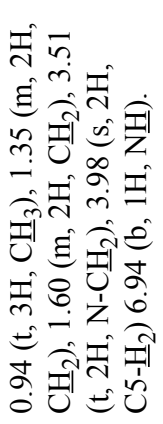 & 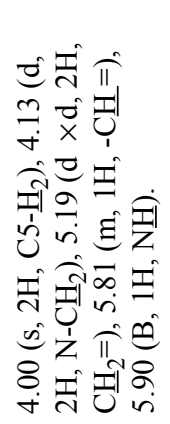 & 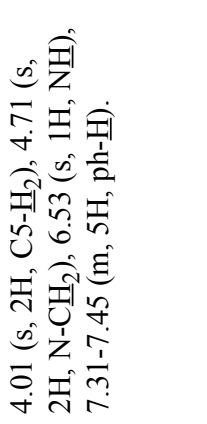 \\
\hline 胥 & స్ల & $\underset{\sim}{\stackrel{్}{\sim}}$ & \্ণ & 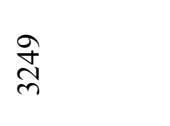 & 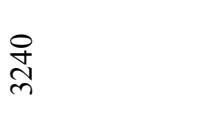 \\
\hline च & 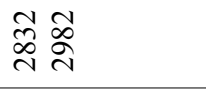 & 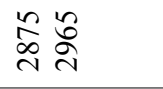 & $\begin{array}{l}\infty \\
\stackrel{\infty}{\infty} \\
\text { ì }\end{array}$ & 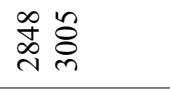 & 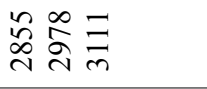 \\
\hline $\begin{array}{l}0 \\
\text { un }\end{array}$ & $\stackrel{ }{\stackrel{2}{I}}$ & बે욜 & क्षे & 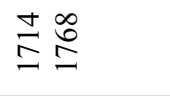 & 도 \\
\hline 这 & సี & ते & $\ddot{N}$ & $\bar{\sim}$ & $\stackrel{\sim}{N}$ \\
\hline
\end{tabular}




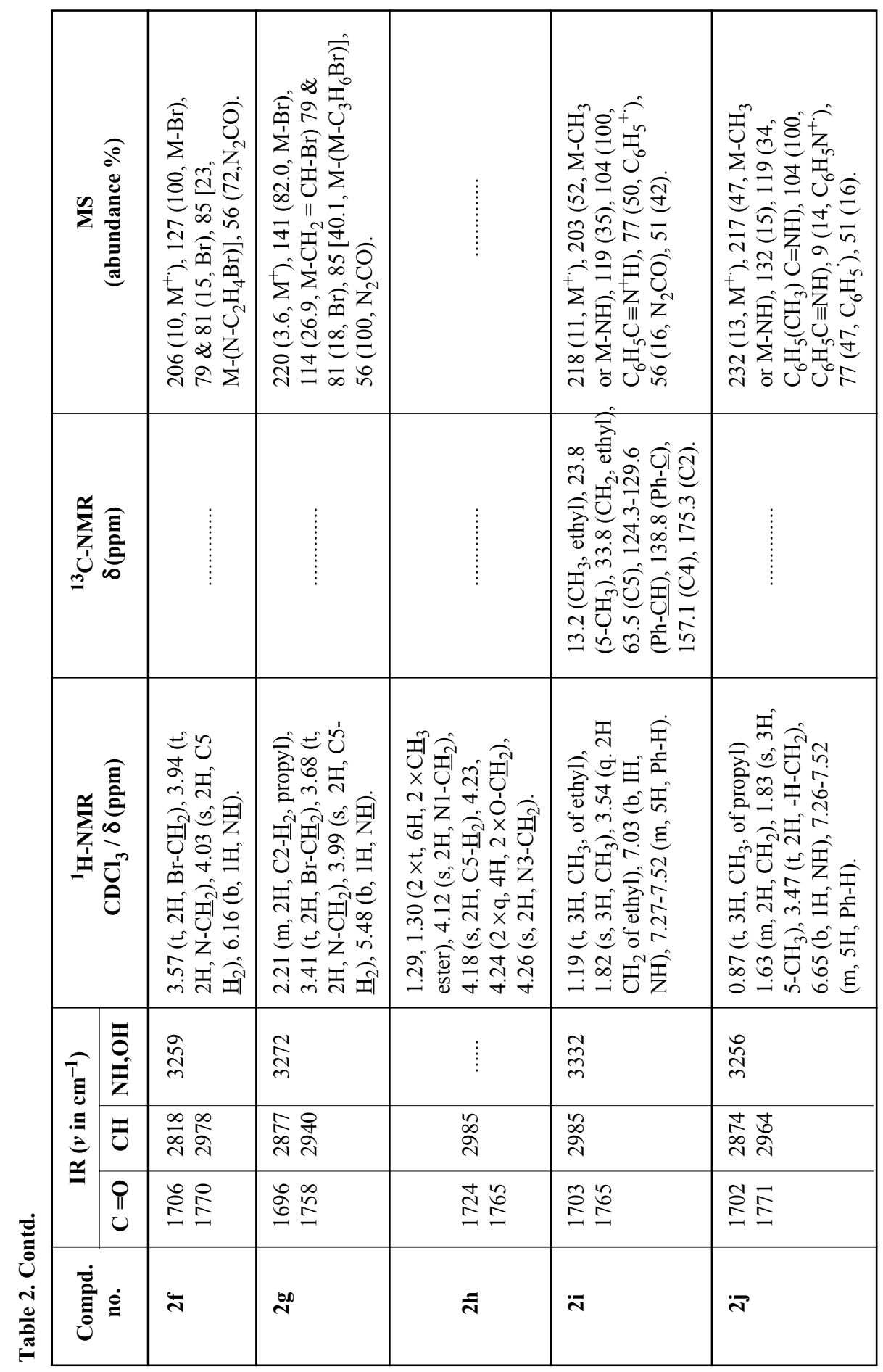




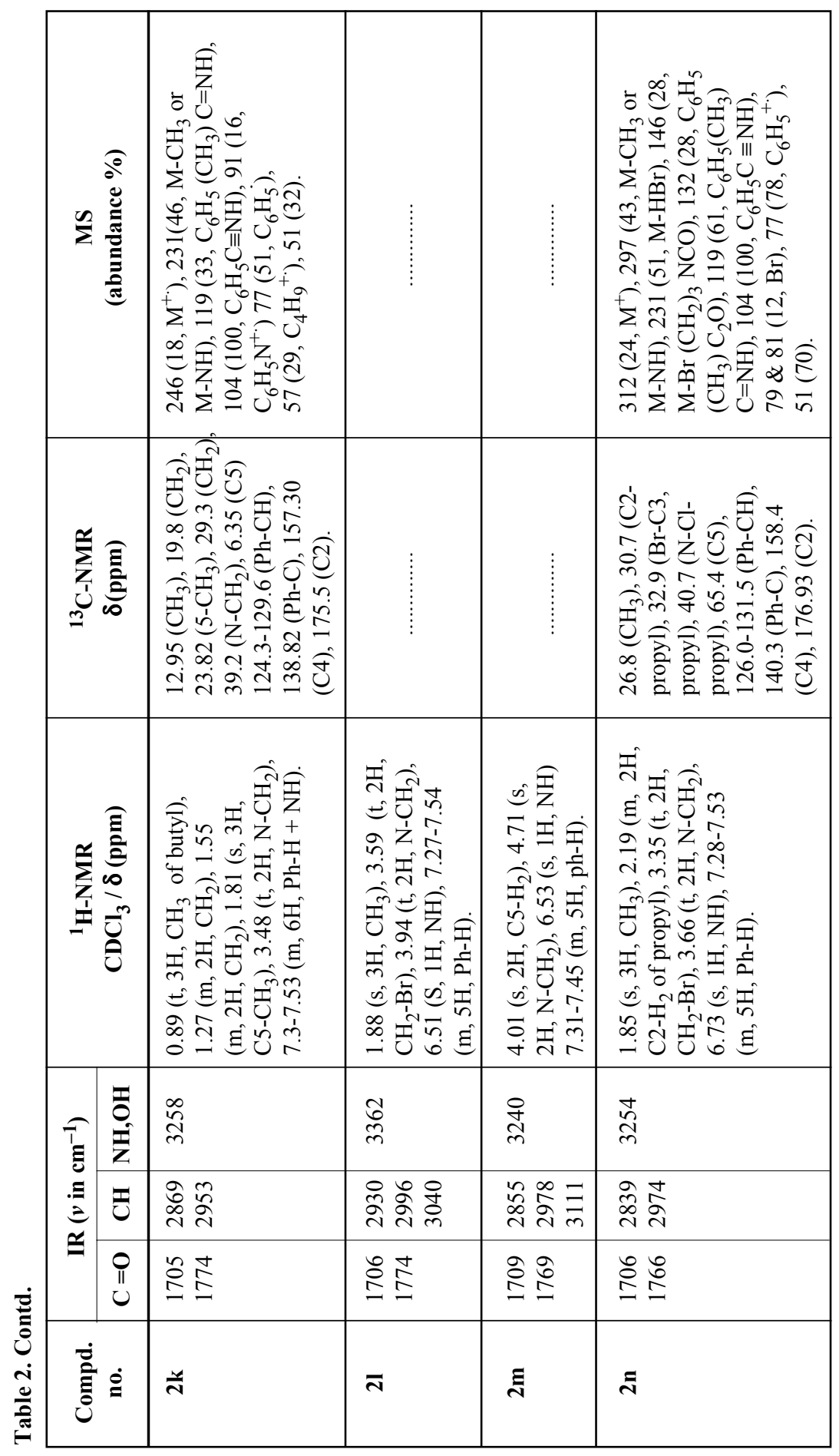




\begin{tabular}{|c|c|c|c|c|}
\hline \multicolumn{2}{|c|}{ 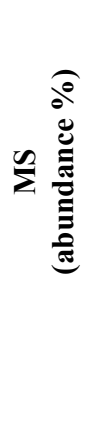 } & 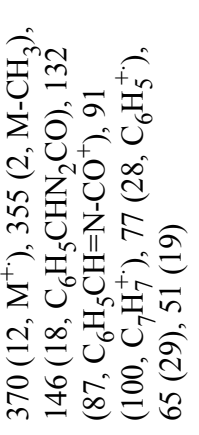 & 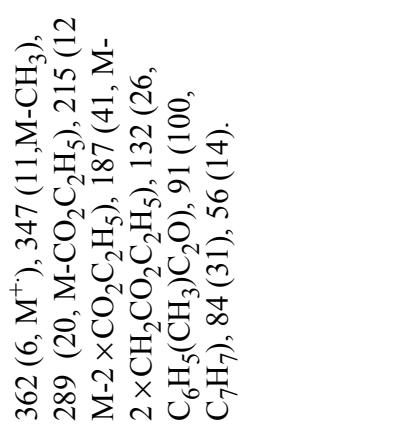 & \\
\hline \multicolumn{2}{|c|}{ 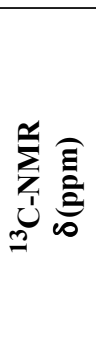 } & 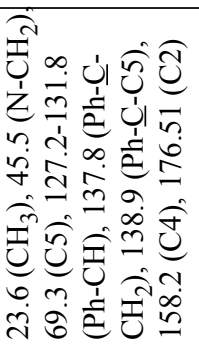 & 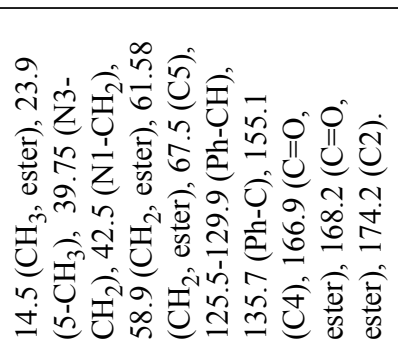 & 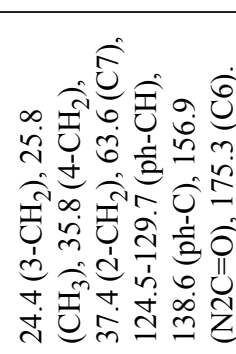 \\
\hline \multicolumn{2}{|c|}{ 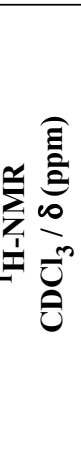 } & 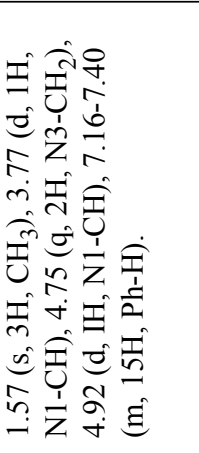 & 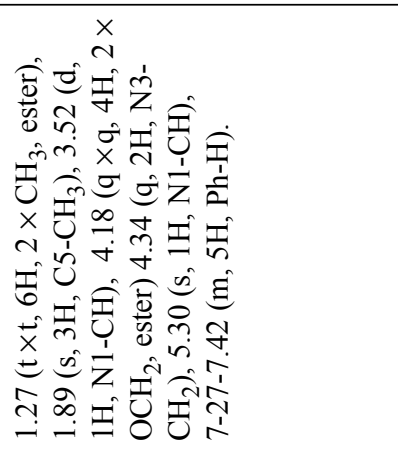 & 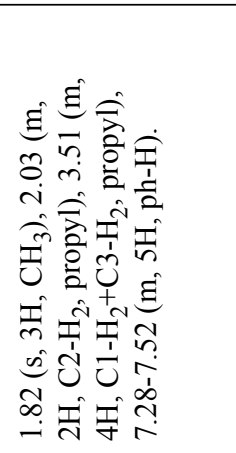 \\
\hline & 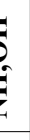 & & $\vdots$ & $\vdots$ \\
\hline & & ले $\overline{\hat{~}}$ & 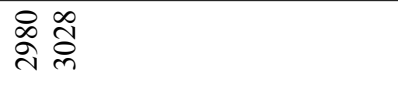 & 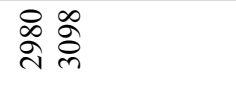 \\
\hline & & 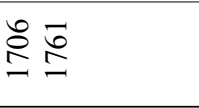 & તి & 오ㄹㅗㅛ \\
\hline \multicolumn{2}{|c|}{ 苞 } & $\stackrel{\sim}{i}$ & సे & $m$ \\
\hline
\end{tabular}


On the other hand alkylation of hydantoin (1a) by n-propyl bromide or allyl bromide (1:5 molar ratio, respectively) under the same PTC reaction conditions and in the presence of $\mathrm{CS}_{2}$ with efficient stirring and TLC-reaction was monitored by TLC, this afforded after 2h, 5-[bis(alkylthio) methylene]-1,3-dialkylimidazolidine-2,4-dione $(\mathbf{4 a}, \mathbf{b})$ in $32 \%$ yield. There is no improvement of the yield of 4 even after 3 days reaction period. Meanwhile, alkylation of hydantoin (1a) by ethyl bromoacetate under the same PTC-reaction conditions and in the presence of $\mathrm{CS}_{2}$ yielded diethyl 2,2'-[[3-(2-ethoxy-2-oxoethyl)-2,5-dioxoimidazolidin-4-ylidene]-methylene]bis (thio)] diacetate (4c) as yellow viscous oil. The products 4 and the unreacted hydantoin (1a) were isolated by column chromatography using diethyl ether/petroleum ether (2:1) (Scheme 3).

The reaction proceeds with nucleopilic addition of the intermediate C5carbonion on $\mathrm{CS}_{2}$ to give the intermediate dithiolate anion which is dialkylated at $\mathrm{N}-1$ and $\mathrm{N}-3$ by n-propyl / or allyl bromide, while N-1 alkylation's, only, by ethyl bromoacetate (Scheme 3).

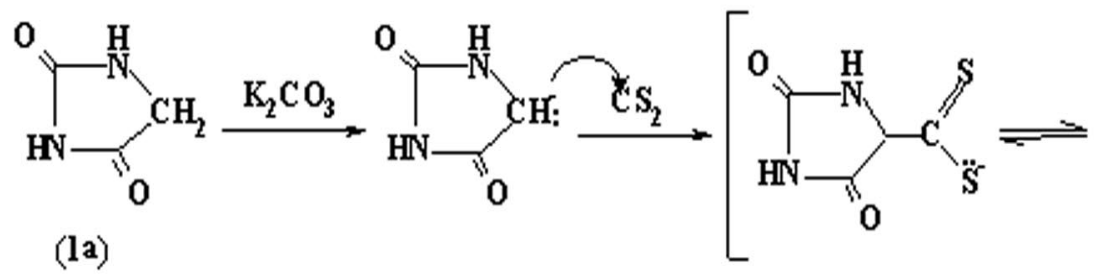<smiles>[R]SC(S[R])=C1C(=O)N([R])C(=O)N1[R]</smiles>

a) $\mathrm{R}: \mathrm{S}, \mathrm{N} 1, \mathrm{~N} 3=\left(\mathrm{CH}_{2}\right)_{2} \mathrm{CH}_{3}$

b) $\mathrm{R}: \mathrm{S}, \mathrm{N} 1, \mathrm{~N} 3=\mathrm{CH}_{2}=\mathrm{CHCH}_{2}$

c) $\mathrm{R}: \mathrm{S}, \mathrm{N} 3=\mathrm{CH}_{2} \mathrm{CO}_{2} \mathrm{C}_{2} \mathrm{H}_{5} ; \mathrm{RN} 1=\mathrm{H}$

Scheme 3

The structure of alkylatedhydantoins (4a-c) has been established by spectral data (Table 3) and elemental analysis (Table 1). 
Table 3. Spectral data of compounds of alkylatedhydantoin (4a-c).

\begin{tabular}{|c|c|c|c|c|c|}
\hline \multirow{2}{*}{$\begin{array}{c}\text { Compd. } \\
\text { no. }\end{array}$} & \multicolumn{3}{|c|}{$\operatorname{IR}\left(\mathrm{v}\right.$ in $\left.\mathrm{cm}^{-1}\right)$} & \multirow{2}{*}{$\begin{array}{c}{ }^{1} \mathrm{H}-\mathrm{NMR} \\
\mathrm{CDCl}_{3} / \delta(\mathrm{ppm})\end{array}$} & \multirow{2}{*}{$\begin{array}{c}\text { MS } \\
\text { (abundance \%) }\end{array}$} \\
\hline & $\mathbf{C}=\mathbf{O}$ & CH & NH & & \\
\hline $4 a$ & $\begin{array}{l}1713 \\
1787\end{array}$ & $\begin{array}{l}2872 \\
2959\end{array}$ & ....... & $\begin{array}{l}0.76\left(\mathrm{t}, 3 \mathrm{H}, \mathrm{CH}_{3}\right), 0.9\left(\mathrm{t}, 3 \mathrm{H}, \mathrm{C}_{3}\right), 1.0 \\
\left(\mathrm{t}, 3 \mathrm{H}, \mathrm{C} \underline{\mathrm{H}}_{3}\right), 1.04\left(\mathrm{t}, 3 \mathrm{H}, \mathrm{C} \underline{\mathrm{H}}_{3}\right), 1.45 \\
\left(\mathrm{~m}, 2 \mathrm{H}, \mathrm{CH}_{2}\right), 1.73\left(\mathrm{~m}, 6 \mathrm{H}, 3 \times \mathrm{CH}_{2}\right), \\
2.91\left(\mathrm{t}, 2 \mathrm{H}, \mathrm{S}-\mathrm{C} \underline{\mathrm{H}}_{2}\right), 3.05(\mathrm{t}, 2 \mathrm{H}, \mathrm{S}- \\
\mathrm{CH}), 3.34\left(\mathrm{t}, 2 \mathrm{H}, \mathrm{N}-\underline{\mathrm{H}}_{2}\right), 3.86 \\
\left(\mathrm{t}, 2 \mathrm{H}, \mathrm{N}-\mathrm{CH} \underline{\mathrm{H}}_{2}\right) .\end{array}$ & $\begin{array}{l}345\left(2, \mathrm{M}^{+\cdot}\right), 260(13, \mathrm{M}-2 \\
\left.\times \mathrm{C}_{3} \mathrm{H}_{7}\right), 226(16), 218 \\
\left(45, \mathrm{M}-3 \times \mathrm{C}_{3} \mathrm{H}_{7}\right), 18(16), \\
175(10), 144(16), 56(100, \\
\left.\mathrm{N}_{2} \mathrm{CO}\right) .\end{array}$ \\
\hline $4 b$ & $\begin{array}{l}1720 \\
1793\end{array}$ & $\begin{array}{l}2857 \\
2983 \\
3082\end{array}$ & ....... & $\begin{array}{l}3.60\left(\mathrm{~d}, 2 \mathrm{H}, \mathrm{S}-\mathrm{C}_{2}\right), 3.69(\mathrm{~d}, 2 \mathrm{H}, \\
\left.\mathrm{S}-\mathrm{C} \underline{\mathrm{H}}_{2}\right), 3.98\left(\mathrm{~d}, 2 \mathrm{H}, \mathrm{N} 1-\mathrm{C}_{2}\right), 4.53 \\
\left(\mathrm{~d}, 2 \mathrm{H}, \mathrm{N} 3-\mathrm{CH}_{2}\right), 5.04-5.36(\mathrm{~m}, 8 \mathrm{H}, \\
\left.4 \times \mathrm{CH}_{2}=\right), 5.63(\mathrm{~m}, 1 \mathrm{H},-\mathrm{CH}=), \\
5.86(\mathrm{~m}, 2 \mathrm{H}, 2 \times \mathrm{CH}=), 5.95-(\mathrm{m}, \\
1 \mathrm{H},-\mathrm{CH}=) .\end{array}$ & \\
\hline $4 c$ & $\begin{array}{l}1722 \\
1783\end{array}$ & 2982 & 3255 & $\begin{array}{l}1.29\left(\mathrm{t}, 9 \mathrm{H}, 3 \times \mathrm{C}_{3}\right), 4.08(\mathrm{~s}, 4 \mathrm{H}, 2 \\
\left.\times \mathrm{S}-\mathrm{C} \underline{\mathrm{H}}_{2}\right), 4.22\left(\mathrm{q}, 6 \mathrm{H}, 3 \times \mathrm{O}-\mathrm{CH}_{2}\right) \\
4.37\left(\mathrm{~s}, 2 \mathrm{H}, \mathrm{N} 3-\mathrm{CH}_{2}\right), 5.79(\mathrm{~b}, 1 \mathrm{H}, \\
\text { N1- } \underline{\mathrm{H}}) .\end{array}$ & .......... \\
\hline
\end{tabular}

\section{References}

[1] Dehmlow, E.V. and Dehmlow, S.S., Phase Transfer Catalysis, VCH Publisher, New York, USA (1983).

[2] Stark, C.M., Liotta, C. and Halpern, M., Phase Transfer Catalysis, Fundamentals, Application and Industrial Perspectives, Chapman \& Hall, New York, USA (1994).

[3] Sasson, Y. and Neumann, R., Handbook of Phase Transfer Catalysis, Chapman \& Hall, New York, USA (1997).

[4] Weber, W.P. and Gokel, G.W., Phase-Transfer Catalysis in Organic Chemistry, Springer, New York, USA (1977).

[5] Freedman, H.H., Pure Appl. Chem., 58: 857 (1986).

[6] Hassan, M.A., Mohamed, M.M., Shiba, S.A. and Khalil, A., Phosphorus Sulfur and Silicon, 157: 97 (2000).

[7] Hassan, M.A., Mohamed, M.M., Shiba, S.A., Abou El-Regal, M.K. and Khalil, A., Phosphorus, Sulfur and Silicon, 178: 1497 (2003).

[8] Hassan, M.A. and Döpp, D., Heterocycles, 45: 451 (1997).

[9] Hassan, M.A., Mohamed, M.M., Shiba, S.A. and Khalil, A., Phosphorus, Sulfur, and Silicon, 158: 91 (2000).

[10] Khalil, A.K., Hassan, M.A., Mohamed, M.M. and El-Sayed, A.M., Phosphorus, Sulfur, and Silicon, 180: 479 (2005).

[11] Karolacawojcie, C.J., Kwait, K.W. and Kieckonono, K., Pharmazie, 50: 114 (1995).

[12] Brouillette, W.J., Jestkov, V.P., Brown, M.L. and Akhtar, M.S., J. Med. Chem., 37: 3289 (1994). 
[13] Miller, K.E., Carpenter, J.F. and Brooks, R.R., Cardiovasc. Drugs Ther., 12: 83 (1998).

[14] Edmunds, J.J., Klutchko, S., Hamby, J.M., Bunker, A.M., Connolly, C.J.C., Winters, R.T., Quin, J., Sircar, I. and Hodges, J.C., J. Med. Chem., 38: 3759 (1995).

[15] López, C.A. and Trigo, G.G., Adv. Heterocycl. Chem., 38: 224 (1985).

[16] Marton, J., Enisz, J., Hosztafi, S. and Timár, T., J. Agric. Food Chem., 41: 148 (1993).

[17] Kieć-Kononowicz, K. and Szymańska, E., Il Farmaco, 57: 909 (2002).

[18] El-Barbary, A.A., Khodair, A.I., Pedersen, E.B. and Nielsen, C., J. Med. Chem., 37: 73 (1994).

[19] Khodair, A.I., El-Subbagh, H.I. and El-Emam, A.A., Bull. Chim. Farm., 136: 561 (1997).

[20] Al-Obaid, A.A., El-Subbagh, H.I., Khodair, A.I. and El-Mazar, M.M., Anti-Cancer Drugs, 7: 873 (1996).

[21] Che'rouvrier, J., Carreaux, F. and Banzureau, J.P., Tetrahedron Lett., 43: 8745 (2002). 


$$
\text { ألْكَلَة هـيدانتوين و 0-مثيل -0 - تحنيل هيدانتوين }
$$

طارق رشاد سبحي ، ومحمد علي حسن ، وحسين أحمد مصطفى ،

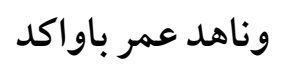

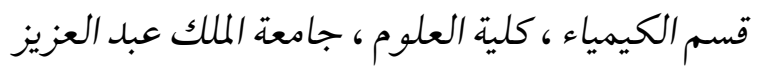

جـــة - المملكة العربية السعودية

المستخلص. تـم ألكلة كل من هـيدانتوين (1a) و ه-مثيل -ه -فنيل

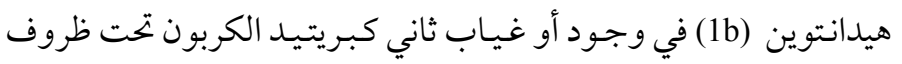

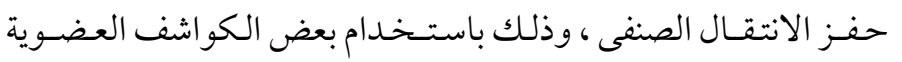

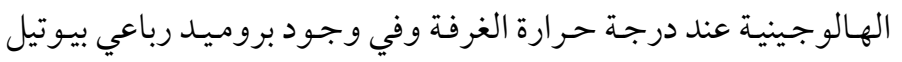

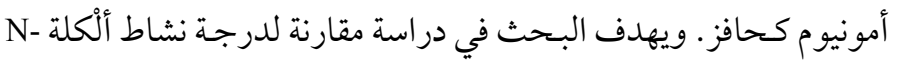

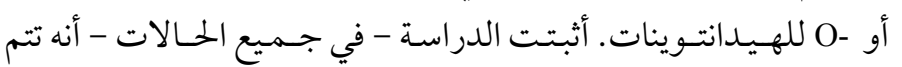

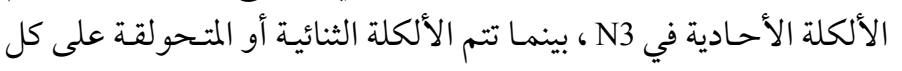

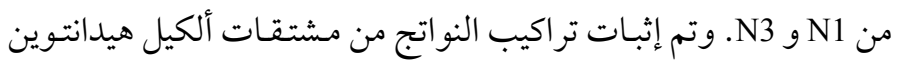

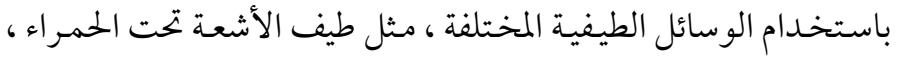

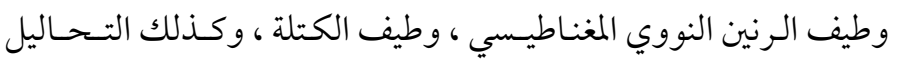

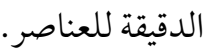

